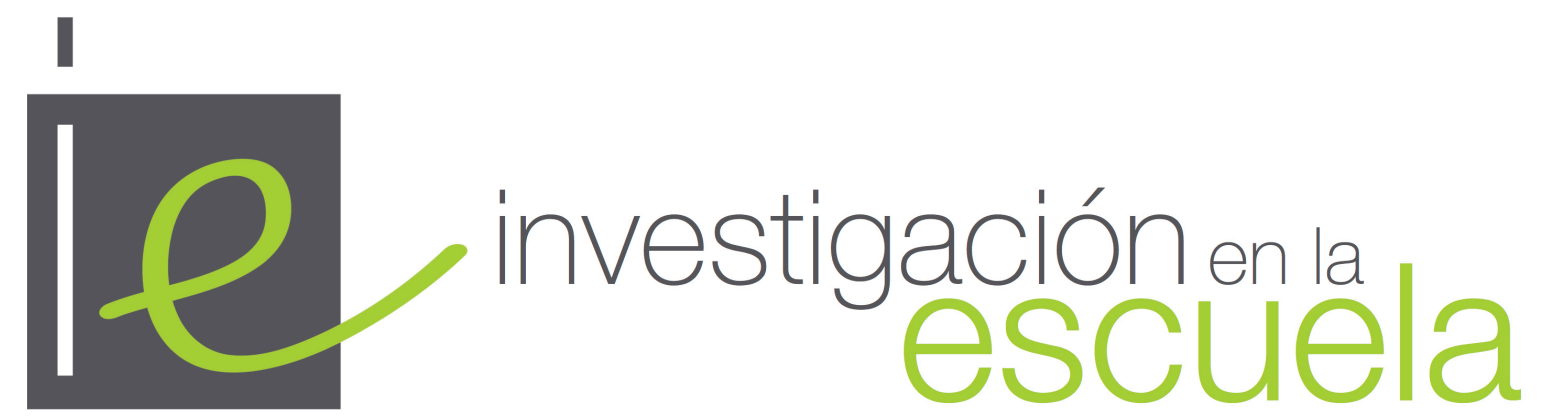

Revista internacional de investigación e innovación educativa

\title{
La educación literaria: una experiencia didáctica de animación a la lectura promovida por el alumnado
}

\author{
Blanca Hernández Quintana \\ Universidad de Las Palmas de Gran Canaria \\ España
}

Citación: Hernández Quintana, B. (2018). La educación literaria: una experiencia didáctica de animación a la lectura promovida por el alumnado. Investigación en la Escuela, 94, 16-30. Recuperado de: http://www.investigacionenlaescuela.es/articulos/R94/R94-2

Resumen: Este artículo describe y analiza una experiencia de aula promovida por el propio alumnado. Se trata de una propuesta didáctica de animación a la lectura, colaborativa e interdisciplinar, en la que participan todas las etapas: Bachillerato, ESO, Educación Primaria y Educación Infantil. El proyecto se desarrolla desde la asignatura de Lengua castellana y Literatura e incluye a las áreas de Educación Plástica, Música e Informática. Se muestran los criterios de diseño y análisis de secuencias y se concluye que el enfoque participativo e interdisciplinar promueve los hábitos de lectura entre los estudiantes.

Palabras clave: "Educación literaria"; "animación a la lectura"; "didáctica"; "proyecto interdisciplinar"; "literatura".

Literary education: a didactic experience of reading motivation promoted by students Abstract: This article describes a classroom experience promoted by students themselves. This reading motivation proposal, which involves primary and secondary school. is based on a collaborative learning. The project is developed from Spanish Language and Literature subject including Plastic Education, Music and Computer Science areas. Project activities and schedule are included in the article and it is concluded that an interdisciplinary and collaborative approach forges reading habits among students. 
Key words: "Literary education"; "reading motivation"; "didactics"; "interdisciplinary project"; "literature".

\section{Éducation littéraire: une expérience didactique d'animation à la lecture promue par les étudiants}

Resumè: Cet article décrit et analyse une expérience de classe promue par les élèves eux-mêmes. Il s'agit d'une proposition didactique, collaborative et interdisciplinaire pour inciter à la lecture, à laquelle participent tous les niveaux d'enseignement: primaire, secondaire et supérieur. Le projet est développé à partir de la matière "Langue et Littérature espagnole" et comprend les domaines des arts plastiques, de l'éducation musicale et del'informatique. Les critères de conception et d'analyse des séquences sont présentés et l'on conclut que l'approche participative et interdisciplinaire favorise les habitudes de lecture chez les étudiants.

Mots-clés: "Éducation littéraire"; "incitation à la lectura"; "didactique"; "projet interdisciplinaire"; "littérature".

\section{Introducción}

“PPara qué sirve esto?”, comentan los alumnos/as. Al empezar el curso, preguntamos al alumnado de cuarto de la ESO qué opinan de la literatura, y esa suele ser su primera respuesta, y añaden que no entienden por qué tienen que memorizar tantos datos de los autores y características de los movimientos literarios: "es un rollo". Además, no suelen relacionar la literatura con los libros. Los perciben como dos compartimentos aislados que, en ocasiones, se encuentran: "en la materia de literatura nos mandan a leer unos libros aburridos que no entendemos para nada". Entonces les preguntamos qué opinan de la lectura, de los libros: la mayoría del alumnado de Secundaria reconoce que no lee, que leer le resulta aburrido, "leer es de frikis", dice, que antes sí leía más, ahora, su tiempo libre lo dedica a estar con el móvil o internet.

"Cuando lo que ocurre en el aula se conecta con la vida real logramos que el conocimiento pase de ser una representación a ser una experiencia, de ser algo ajeno a ser algo nuestro" (Acaso, 2014, p. 17). De alguna forma, estas palabras sintetizan el proyecto que hemos realizado en las aulas, un proyecto basado en una experiencia de aprendizaje real.

De estas reflexiones surgieron las orientaciones didácticas dirigidas al desarrollo de la educación literaria. La realidad está hecha de palabras que dan forma a nuestro pensamiento y que, a su vez, nos definen. Sin el registro lingüístico apropiado la realidad se empobrece y nuestro pensamiento también. Dominarlas significa desarrollar un discurso propio que nos permite comprender y construir el mundo que nos rodea, y formar nuestro pensamiento, y a partir de ahí cambiarlo y mejorarlo. No proporcionar estas herramientas a nuestro alumnado implica no sólo darles una formación incompleta, sino también ponerlos en situación de desventaja frente a otros que sí las dominan. Como comenta Millás (2016), resulta difícil cuantificar o medir, de alguna forma, los beneficios que proporciona la lectura, porque son intangibles, no medibles, pero los estudiantes constatan que, después de leer un libro, saben más, conocen un poco mejor el mundo que les rodea y a ellos mismos, sin saber explicar por qué, como si sólo los libros pudieran darnos acceso a una parcela de la realidad a la que no podríamos acceder de otro modo.

En este marco, este trabajo desarrolla una propuesta didáctica de animación a la lectura, colaborativa e interdisciplinar, en la que participan todas las etapas: Bachillerato, ESO, Educación Primaria y Educación Infantil. El proyecto se desarrolla desde la asignatura de Lengua castellana y Literatura e incluye a las áreas de Educación Plástica, Música e Informática. A través de la implementación de una serie de actividades, talleres y dramatizaciones basadas en la innovación 
metodológica y donde el alumnado es el protagonista, pretende inculcar el hábito lector y la necesidad del desarrollo del pensamiento crítico en el alumnado.

\section{Marco teórico}

Desde hace décadas se habla de la necesidad de la renovación de la didáctica de la literatura. Quizá las respuestas del alumnado son la consecuencia de una metodología aferrada al método historicista, que se sustenta en una secuenciación cronológica de contenidos, datos, movimientos literarios, rasgos estilísticos, etc. Es evidente que son necesarios determinados conocimientos para poder comprender un texto, o el hecho literario, según explica Mendoza Fillola (2004), pero es imprescindible que la didáctica y la metodología docentes se orienten en despertar la curiosidad en el alumnado, y no en un mecánico ejercicio de memorización e interpretaciones unilaterales. A este rechazo a la literatura y los libros, contribuye, también, el hecho de haber unido las materias de Lengua y Literatura en una sola asignatura, siendo dos disciplinas totalmente diferentes, que se complementan, sí, pero son diferentes. La consecuencia, como comenta Enkvist (2011, p. 239), es que el texto escolar se hace "más breve pero tan denso, como un texto de enciclopedia", lo que ha convertido en más difícil su comprensión para el alumnado. Asimismo, Colomer (2010) apunta que se ha ido reduciendo la presencia de los textos a favor del conocimiento informativo para abarcar toda la historia de la literatura.

Si el estructuralismo, en su fin último, niega al sujeto y convierte la estructura en la piedra angular de la comprensión, el postestructuralismo profundiza en la deconstrucción del texto como una nueva práctica de lectura. Roland Barthers (2002) involucra al lector en la literatura, y defiende la multiplicidad de visiones del texto literario. El centro de su interés es el lector y el acto de leer y distingue entre textos legibles y escribibles, siendo los primeros aquellos que no proponen del lector más que la recepción o el rechazo del texto, y, por el contrario, los textos escribibles son los que le permiten crear, involucrarse, es decir, que podrían ser re-escritos. Y siguiendo estos postulados hemos diseñados nuestro proyecto didáctico.

Asimismo, las teorías feministas postestructuralistas explican los mecanismos económicos, políticos y sociales que mantienen el conocimiento de tipo sexista y patriarcal. Muchos textos disfrazan de normalidad historias impregnadas de la ideología de la superioridad masculina, la invisibilidad femenina o encasillan a hombres y mujeres en un rol predeterminado. Además, como apunta Servén (2008), el sistema educativo sigue enseñando a leer y a analizar los textos con unos objetivos determinados por unos programas educativos diseñados a partir del canon institucional en el que no aparecen escritoras y los personajes femeninos suelen aparecer estereotipados. Por eso, proponemos la educación literaria como un espacio desde el que avanzar hacia el desarrollo de identidades no sexistas e igualitarias.

Hace años que la teoría de la recepción puso el énfasis en el lector ante el hecho literario, proponiendo diferentes enfoques, pero sigue faltando la sistematización de un método. Más allá de las teorías literarias, críticas, etc., consideramos necesario abrir nuevos enfoques y sugerencias que enriquezcan la comunicación y la lectura. Broitman (2015) incide en que cuando el lector se apropia de la obra, saca de ella nuevas preguntas y provoca otras respuestas y otros textos. De alguna manera, "supone un reto para la cultura del consumo instantáneo. El lector se ve obligado a descifrar el significado [...] como si autor y lector se convirtieran en cocreadores de la obra" (Eagleton, 2016, p. 143), y desde estas perspectivas cobra relevancia el papel activo del alumnado en las clases de literatura.

Quizá es una forma de cuestionar el hecho pedagógico o, según se mire, una manera de explotar las posibilidades del currículum en aras de la innovación y la investigación, elementos 
fundamentales en la labor docente, que debe ir más allá del libro de texto. No solo el alumnado, el profesorado debe tener también un papel más activo en la aplicación del currículum, considerado como un sistema abierto y flexible, y debe convertirse en creador y contagiar esa creatividad a los estudiantes ya que, por ejemplo, difícilmente un profesor/a que no sea lector podrá desarrollar el hábito lector en su alumnado.

La reorientación de la didáctica de la literatura pasa por concretar el enfoque y, sobre todo, por delimitar unos objetivos específicos que conllevan la necesidad de implementar la educación literaria en las aulas, que, como expone Mendoza Fillola (2004), consiste en enseñar a participar en el proceso de recepción, en la actualización interpretativa del discurso literario y en formar lectores. "El verbo leer no soporta el imperativo" (Pennac, 1993), así pues, debemos emplear la motivación como uno de los principales métodos para que leer vuelva a ser un placer para el alumnado. Si se reconocen en las lecturas, habremos conseguido que los libros dejen de ser algo abstracto y ajeno, que se sientan lectores activos, y como indica O'Connor (2016), que como lectores establezcan relaciones con el texto a partir de las cosas que le son mostradas para llegar a comprender e interpretar su significado. En el desarrollo de la educación literaria, la metodología, el criterio de selección de textos y la integración de la teoría de la recepción, juegan un papel fundamental. Nuestro proyecto va encaminado a armonizar estas ideas.

\section{Metodología de la investigación}

\section{Contextualización}

Durante los cursos 2015/16 y 20016/17, el alumnado del colegio Salesianos de Las Palmas de Gran Canaria desarrolla este proyecto de animación a la lectura. El centro tiene tres edificios, uno para Educación Infantil, otro para Primaria, y otro para Secundaria y Bachillerato. Cuenta con tres líneas por curso, con una media de 30 alumnos/as, aproximadamente, por aula.

El proyecto se desarrolla desde la asignatura de Lengua castellana y Literatura de cuarto de la ESO e intervienen, también, los cursos de primero de Primaria, Infantil de tres años y primero de Bachillerato. Se articula con la materia de Literatura de cuarto de la ESO, desde la perspectiva didáctica de la formación y la educación literaria, y se llevan a cabo actividades de animación a la lectura, lecturas de diferentes libros, debates sobre los libros, creación de textos propios, prácticas de dramatización en clases de diferentes cursos y propuestas de actividades para animar a la lectura elaboradas por el alumnado.

Asimismo, se imbrica con el trabajo interdisciplinar de diferentes áreas como Educación Plástica y Visual, que se encarga de guiar al alumnado en la elaboración del decorado, disfraces y atrezo; Música colabora en la creación de las melodías para las dramatizaciones de los cuentos; y los alumnos/as de cuarto de ESO ponen en práctica los programas aprendidos en las clases de Informática para elaborar vídeos de animación a la lectura que se proyectan a los de Primaria el día de la dramatización.

El proyecto final culmina con la celebración del Día del Libro, que es el día en que se realizan las dramatizaciones: el alumnado de cuarto de la ESO trabaja diferentes cuentos a lo largo del curso para luego hacer una dramatización en el aula al alumnado de primero de Educación Primaria. Luego, los alumnos/as de primero de Educación Primaria dramatizan los mismos cuentos a los de Educación Infantil y a los de cuarto de la ESO. Pero, quizá, la celebración del Día del libro es tan solo el colofón de una experiencia con trabajos en el aula que se desarrollan durante todo el curso escolar, unos trabajos para los que se han diseñado una metodología y unas sesiones específicas. 


\section{Metodología}

La necesidad de la innovación didáctica y educativa que precisa la materia de Literatura pasa por un proceso de renovación metodológica que posibilite la formación de lectores/as. Asimismo, como explican Sánchez Calleja y Sánchez Román (2015), se trata de poner el énfasis en lo que el alumnado trae en el presente cotidiano, sin menoscabo de una estructura de contenidos y de un modelo de intervención, por eso hemos utilizado diferentes metodologías.

Una metodología expositiva para explicar ciertos conceptos e ideas, enfocada, a su vez, a formularles preguntas y a fomentar el debate. Una segunda metodología que se sustenta en el aprendizaje significativo, fundamentada en una experiencia real para el alumnado. Y, por último, trabajo cooperativo mediante el desarrollo de situaciones de aprendizaje que posibiliten la interacción del alumnado y su corresponsabilidad en la planificación de su propio trabajo. La metodología que hemos empleado pretende trazar un puente que una la vida de los personajes literarios con la de nuestro alumnado.

Con esta metodología, el alumnado siente que el proceso de aprendizaje tiene una trascendencia, un alcance real y comprueba que las historias que cuentan los libros tienen su correlato en la vida real: las palabras pasan de la página al pensamiento y de ahí a un contexto inmediato y real. Sirven para algo, porque tienen una función real en la sociedad. Como comenta Abarca Alpízar (2016), la metodológica participativa y procesual responde al pensamiento complejo, que propone nuevas miradas al conocimiento de la realidad, de la vida, y que integran procesos teóricos, prácticos y conceptuales a través de diferentes acciones. Por lo que consideramos fundamental dividir el proyecto en una serie de sesiones donde se trabajan la literatura y los libros como una experiencia participativa que destierra la idea de que "leer no sirve para nada".

\section{Objetivos: estándares de aprendizaje}

Entre otros, el planteamiento curricular del área de Lengua castellana y Literatura incide en el desarrollo de estrategias de comprensión lectora y en la educación literaria —entendida como la capacidad, gradualmente adquirida, de seleccionar lecturas propias y como el dominio de un conjunto de estrategias para disfrutar y aprender leyendo-. Teniendo en cuenta estas premisas, hemos reflexionado sobre la idea del currículo como una construcción abierta, en proceso de elaboración, que nunca se acaba para, de este modo, permitir que la sorpresa y la espontaneidad entren en las aulas, y permitan que surjan nuevas ideas derivadas no solo de cuestiones personales, sino también de las de todos los agentes que forman parte del aula: el profesorado, los compañeros/as, los libros, etc. Los objetivos se corresponden con los estándares de aprendizaje del Bloque 4. Educación literaria (Real Decreto 1105/2014, de 26 de diciembre, 2015, p. 202) y son los siguientes:

- Fomentar y consolidar el hábito lector.

- Aprender estrategias de animación a la lectura.

- Trabajar la creatividad y la imaginación.

- Aprender a trabajar en grupo: respeto, colaboración, capacidad de escucha, responsabilidad, participación, etc.

- Dramatizar textos creados y adaptados por ellos/as.

- Favorecer la autonomía y la confianza en sus capacidades.

- Desarrollar y mejorar la capacidad lingǘstica, comunicativa, expresiva, emocional y creativa.

- Incentivar el sentido crítico y la iniciativa personal.

- Fomentar diferentes tipos de lenguaje en el aula: textual, visual, musical, artístico, etc.

- Convertir el proceso de aprendizaje en una experiencia vivencial. 


\section{Temporalización}

El Proyecto comienza a desarrollarse en noviembre, y en los meses de septiembre y octubre vamos familiarizando al alumnado con nuevas dinámicas de cómo trabajar y de estar en el aula. Por ejemplo, los viernes, día de lectura, se transforma el aula en un lugar acogedor para leer: se traen cojines y se acuestan a leer en el suelo, encima de las mesas, en el alféizar de la ventana, etc. Si el tiempo lo permite bajamos a leer en las canchas o en el patio del recreo. Las sesiones tienen lugar cada viernes según el calendario programado. En noviembre se comienza con las estrategias de animación a la lectura, la lectura de los libros, comentarios y debates en clase sobre su contenido. Se eligen los libros que van a adaptar y a dramatizar. En diciembre, se forman los grupos, se reparten los libros y se comienza a adaptar el relato: si es oportuno se cambia el vocabulario, los diálogos, el final, se define la caracterización de los personajes, se concretan los espacios y se realiza el listado de materiales necesarios. Comienzan en enero los ensayos de la dramatización y se compagina con la construcción de materiales, decorados, disfraces, etc. En febrero, se incorporan los alumnos/as de Bachillerato a los ensayos para poner música a cada cuento. Se intenta que en marzo empiecen a grabar los vídeos de los ensayos, los de animación a la lectura y se preparan estrategias y actividades de animación a la lectura para después de la dramatización. Para finalizar, en abril el alumnado de cuarto de la ESO y los músicos de primero de Bachillerato dramatizan los cuentos a los de Primaria, y en mayo, los alumnos/as de Primaria realizan la dramatización de los mismos cuentos al alumnado de Infantil de tres años y a los de cuarto de la ESO.

\section{Descripción de la experiencia}

El trabajo inicial de este proyecto comienza con la lectura de tres libros. Su elección se vincula con el contenido curricular de la materia de literatura, en concreto con los movimientos literarios que se imparten en cuarto de la ESO en la primera evaluación: Romanticismo, Realismo y Modernismo. Aunque las lecturas están dirigidas al alumnado de Primaria, adaptamos su contenido a la realidad de Primaria y de Secundaria y lo relacionamos con cada movimiento literario en cuestión. El proyecto "Yo cuento, tú cuentas, contamos todos" se ha divido en diferentes sesiones que se desarrollan de la siguiente manera.

Primero, se realiza la lectura en voz alta de los libros seleccionados. Se lleva a cabo siguiendo una serie de estrategias de animación a la lectura: antes de la lectura, a partir del título o del dibujo de la portada, se les pide que adivinen de qué puede tratar el libro, qué les sugiere la portada, etc.; durante la lectura se les solicita que expliquen qué les está haciendo sentir o cómo acabarían la historia; después de la lectura comentan qué les ha parecido el relato, si alguna vez les ha ocurrido algo parecido, qué hubiesen hecho ellos/as en su lugar, etc.

Los libros seleccionados son Enamorados (Dautremer, 2014), Orejas de Mariposa (Aguilar, 2008) y La Princesa Listilla (Cole, 1990). El género narrativo de los tres libros es el cuento corto, escrito en prosa, de unas veinte páginas y con ilustraciones. El lenguaje es sencillo y todos presentan un conflicto que se resuelve al acabar el cuento.

En Enamorados un grupo de niños/as de Primaria se pregunta qué es eso de estar enamorado y cada uno va dando su respuesta, a cuál más disparatada y original. Relacionamos, entonces, su contenido con la temática del Romanticismo: el concepto del amor y del ideal platónico y con los poemas de Bécquer (2006), leídos en las clases de literatura.

Orejas de Mariposa cuenta la historia de una niña que sufre las burlas y el acoso de sus compañeros porque tiene las orejas grandes, porque es pobre y lleva los calcetines rotos, pasa hambre y siempre le suenan las tripas, etc. Vinculamos esta temática con el Realismo: la crítica de los defectos y males que afectan a la sociedad, la denuncia ante la falta de empatía, y lo enlazamos con los fragmentos de Marianela (Pérez Galdós, 2001), leídos en clase de literatura. 
La Princesa Listilla desmonta los cuentos tradicionales sobre princesas pasivas, sumisas y aburridas cuyo único objetivo en la vida es esperar a su príncipe azul para casarse. La protagonista quiere tomar las riendas de su vida y se niega a casarse porque quiere seguir haciendo aquellas cosas que más le gustan. Enlazamos este libro con el Modernismo, en concreto con el poema Sonatina (Darío, 2002), que presenta una princesa triste y aburrida que espera la llegada de su príncipe azul, como único acicate en su vida. Sin desmerecer las aportaciones literarias y estéticas de este movimiento, invitamos a que hagan una lectura crítica sobre su significado y comparen a esta princesa con la princesa listilla.

En la siguiente sesión, elaboran talleres a partir de una idea propuesta que, en algunas ocasiones, se basan en las técnicas ideadas por Rodari (2002). El objetivo es trabajar la creatividad y el pensamiento crítico. Por ejemplo, les pedimos que escriban un texto explicando qué es para ellos/as estar enamorados y su idea sobre las relaciones en pareja, que redacten un texto denunciando los males de la sociedad actual -como el acoso, la discriminación, la violencia, juzgar por la apariencia física, etc.- y que propongan soluciones para erradicarlos o proponemos trabajar la coeducación en el aula a través de la educación literaria: se debate sobre si existe la igualdad entre hombres y mujeres. Aplicando la teoría de la recepción, escuchamos y debatimos sobre las múltiples interpretaciones que aporta el alumnado.

A continuación, se forman tres grupos por aula. En ocasiones, son los propios alumnos/as quienes piden que el profesorado los haga. Se vigila que sean lo más heterogéneos posibles: mismo número de alumnos que alumnas, diferentes perfiles (tímidos, extrovertidos...), diferentes habilidades, etc. Cada grupo elige uno de los tres libros, -si no hay consenso, se hace por sorteo- y comienza la adaptación del cuento. Se les dan algunas indicaciones que pueden seguir: si es necesario, cambiar el vocabulario y adaptarlo al alumnado de Primaria, ampliar, reducir o modificar los personajes atendiendo al número de alumnos/as que hay en el grupo, repartir por igual la participación de todos los componentes del grupo, definir bien los sentimientos, el carácter, las pulsiones de cada personaje así como las características físicas, psicológicas y la indumentaria que van a llevar, delimitar los escenarios, reparto de los personajes y elaborar una lista de los materiales que van a necesitar.

Antes de comenzar los ensayos se les da una serie de pautas para la dramatización, como no dar nunca la espalda al público, exagerar todos los movimientos, proyectar bien la voz y vocalizar adecuadamente, meterse en el personaje, etc.

Cuando terminan los ensayos, con la colaboración del área de Educación Plástica y Visual, comienza la elaboración de materiales: decorado, disfraces, atrezo, etc. Después, ensayan con los materiales para dar más verosimilitud a la dramatización y comprobar si se mueven con soltura con ellos puestos. Luego, se incorpora el alumnado de primero de Bachillerato para ver las obras e incluir música en directo. Con la colaboración del área de Música, crean composiciones musicales para cada cuento adaptadas a la acción: miedo, alegría, misterio, etc. En este último curso, el violín y la guitarra han sido los instrumentos elegidos.

En la siguiente sesión, graban una serie de vídeos de animación a la lectura para proyectarlos el día de la dramatización. Escriben el guion, reparten los personajes, graban las escenas y hacen el montaje, con la colaboración del área de Informática. Los vídeos hablan de los libros: por qué es divertido leer, de qué hablan los libros, qué enseñan, qué hacen sentir, etc. También graban los ensayos y luego los visualizan para corregir posibles errores y mejorar las actuaciones.

Una vez realizadas estas sesiones, preparan estrategias de animación a la lectura para antes, durante y después de la dramatización ante el alumnado de primero de Primaria. Por ejemplo, antes de dramatizar La Princesa Listilla, les preguntan qué es para ellos/as ser una princesa, qué princesas conocen, cómo son, etc. Durante la dramatización, se dirigen al alumnado para preguntarles si creen que los príncipes podrán superar las pruebas que les ha puesto la princesa listilla o si las consideran 
muy difíciles, también animan a su participación: una prueba consiste en tocar la flauta con la nariz y alguien del público sale a intentarlo. Al finalizar, les piden que propongan finales alternativos, que inventen nuevas princesas, etc.

Para finalizar, en el mes de abril, los alumnos/as de cuarto de la ESO A dramatizan en el aula de primero de Primaria A, y lo mismo con los cursos B y C. Los narradores de cada cuento entran en sus aulas correspondientes, montan el decorado y preparan el proyector. Entonces, los narradores se presentan: "Buenos días, hoy estamos aquí los alumnos y alumnas de cuarto de ESO para contarles un cuento. ¿Les gustan los cuentos? ¿Les gusta leer?, etc.”, y proyectan el vídeo dedicado a la animación de la lectura. Una vez acabada la proyección, los narradores hacen preguntas sobre el hábito lector y comienzan las dramatizaciones. La actividad dura las tres primeras horas de la mañana, de 8.00 a 10.45 horas. En mayo, los de primero de Primaria dramatizan los mismos cuentos a los de Infantil de tres años y a los cuartos de Secundaria.

\section{Criterios de evaluación}

La evaluación de las actividades que engloba este proyecto se lleva a cabo mediante una escala de observación, toma de notas sobre las dinámicas de trabajo y el proceso de aprendizaje con una rúbrica en la que figuran los siguientes ítems:

Tabla 1

Criterios de evaluación

\begin{tabular}{ll}
\hline Curso: & $\begin{array}{c}\text { Inadecuado } \\
\text { Poco } \\
\text { adecuado }\end{array}$ \\
\hline Participación & \\
en el aula & \\
Trabajo en \\
grupo \\
Redacción \\
Dramatización \\
Animación \\
Organización \\
Colaboración \\
Habilidades \\
Lingǘsticas \\
Implicación
\end{tabular}

\section{Resultados}

Para valorar los resultados hemos utilizado el método de la triangulación de datos, usado para establecer una valoración en proyectos e investigaciones de enfoque cualitativo, y que comprende el uso de varias estrategias. Esta herramienta de medición nos permite, también, valorar los resultados y la eficacia del proyecto llevado a cabo. Del mismo modo, la triangulación posibilita visualizar, con mayor consistencia, los errores o problemas del proceso y las actividades realizadas en aras de la consecución de los objetivos. En la triangulación hemos contrastado las entrevistas individuales, los grupos de debates o discusión y el cuestionario.

En las entrevistas personales se han recogido valoraciones personales referentes al hábito lector. La mayoría del alumnado de Secundaria comenta que los libros le han hecho pensar en determinadas cuestiones que antes no se habían planteado. Por ejemplo, en Orejas de mariposa, frente 
al acoso que recibe Mara por parte de los compañeros/as de clase, no eran conscientes de cómo el silencio te hace cómplice del acoso o humillación hacia un compañero/a. Algunos reconocieron haber sido testigos de insultos proferidos a otros alumnos/as por su condición sexual o por su físico, pero que habían decidido mantenerse la margen porque no era "asunto mío".

Muchos comparten la idea de que les ha gustado hacer la adaptación al relato, reescribir la historia, cambiar los diálogos, los personajes y el final porque "lo hacían ellos/as". A otros les ha parecido más entretenido la construcción de materiales, los foros de debates o la dramatización. Reconocen que les daba un poco de vergüenza actuar para los de Primaria, y que se apoyaron en el grupo para disimularlo. En general, cuentan que "leer libros así, sí es divertido".

Cuando se pregunta al alumnado de Primaria por estas cuestiones, las respuestas son diferentes. Conciben la lectura como algo divertido y entretenido. Suelen leer a menudo y tienen definidos sus gustos literarios: predominan los cuentos maravillosos y de hadas. Sin embargo, se sienten igual de nerviosos que el alumnado de Secundaria por el hecho de dramatizar el cuento ante los alumnos de Infantil y de la ESO. Para ellos/as es toda una experiencia que, literalmente, les quita el sueño hasta el día de la puesta en escena.

Confrontamos, ahora, las opiniones vertidas por el alumnado con otra herramienta de medición que hemos utilizado: los grupos de debates y discusión. Al finalizar cada libro, se establece un foro de debate que permite escuchar sus opiniones y discutirlas con el grupo. El profesorado hace una serie de preguntas sobre el libro y se establece un foro de discusión donde comparten sus opiniones. Además, en esta actividad utilizamos la técnica de la observación participante y compartimos con el alumnado nuestra experiencia, contexto y vida cotidiana para llegar a descubrir y conocer la información del alumnado sobre su propia realidad y relacionarla con la dinámica de los libros, para que desarrollen la actitud crítica.

Por ejemplo, al leer La Princesa Listilla, explican que les ha ayudado a tomar consciencia de la desigualdad existente entre hombres y mujeres, y de la presencia de estereotipos sexistas en la sociedad actual. Además, algunos alumnos/as pidieron ampliar las sesiones de los ensayos y la de elaboración de materiales porque se vieron muy justos de tiempo.

La tercera recogida de información con la que triangular las valoraciones ha sido a través de un cuestionario. En primero de Primaria, de un total de 81 alumnos/as, se hace la encuesta de manera aleatoria y anónima a 50 , cuyos resultados podemos ver en la tabla 2 y figura 1 :

Tabla 2

Resultados de valoraciones cuestionario

\begin{tabular}{lllllll}
\hline Curso: & Nada & Poco & Regular & Bastante & Mucho Total \\
\hline $\begin{array}{l}\text { Las sesiones me han } \\
\text { gustado }\end{array}$ & 0 & 0 & 0 & 5 & 45 & 50 \\
$\begin{array}{l}\text { He aprendido cosas } \\
\text { nuevas }\end{array}$ & 0 & 0 & 0 & 42 & 8 & 50 \\
$\begin{array}{l}\text { Me ha gustado participar } \\
\text { con niños/as de otros }\end{array}$ & 0 & 0 & 0 & 2 & 48 & 50 \\
$\begin{array}{l}\text { cursos } \\
\begin{array}{l}\text { Dramatizar un cuento } \\
\text { me ha resultado }\end{array}\end{array}$ & 0 & 0 & 0 & 38 & 12 & 50 \\
$\begin{array}{l}\text { estimulante } \\
\text { Ha mejorado mi idea } \\
\text { sobre la lectura }\end{array}$ & 0 & 0 & 0 & 39 & 11 & 50 \\
\hline
\end{tabular}




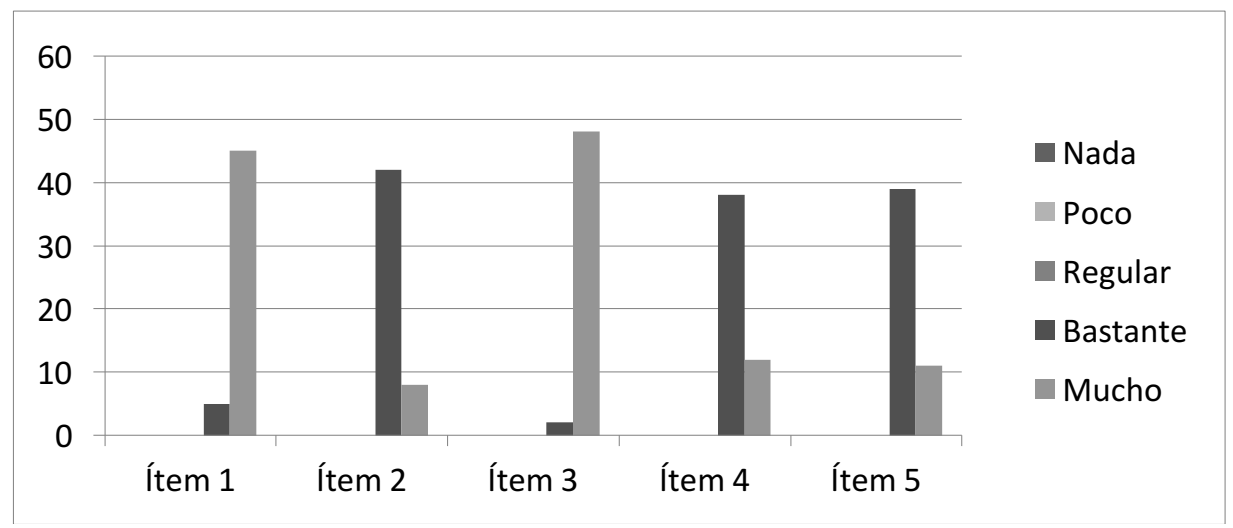

Figura 1. Resultados de valoraciones cuestionario

En cuarto de la ESO, de un total de 94 alumnos/as, se hace la encuesta de manera aleatoria y anónima a 70, cuyos resultados podemos ver en la tabla 3 y figura 2 .

Tabla 3

Resultados encuesta alumnado $4^{\circ}$ ESO

\begin{tabular}{lllllll}
\hline Curso: & Nada & Poco & Regular & Bastante & Mucho & Total \\
\hline $\begin{array}{l}\text { Las sesiones me han } \\
\text { gustado }\end{array}$ & 0 & 1 & 3 & 8 & 58 & 70 \\
$\begin{array}{l}\text { He aprendido cosas } \\
\text { nuevas }\end{array}$ & 0 & 2 & 5 & 18 & 45 & 70 \\
$\begin{array}{l}\text { Me ha gustado participar } \\
\text { con niños/as de otros } \\
\text { cursos }\end{array}$ & 0 & 0 & 0 & 20 & 50 & 70 \\
$\begin{array}{l}\text { Dramatizar un cuento } \\
\text { me ha resultado }\end{array}$ & 0 & 0 & 0 & 49 & 21 & 70 \\
$\begin{array}{l}\text { estimulante } \\
\begin{array}{l}\text { Ha mejorado mi idea } \\
\text { sobre la lectura }\end{array}\end{array}$ & 0 & 0 & 6 & 42 & 22 & 70 \\
\hline
\end{tabular}

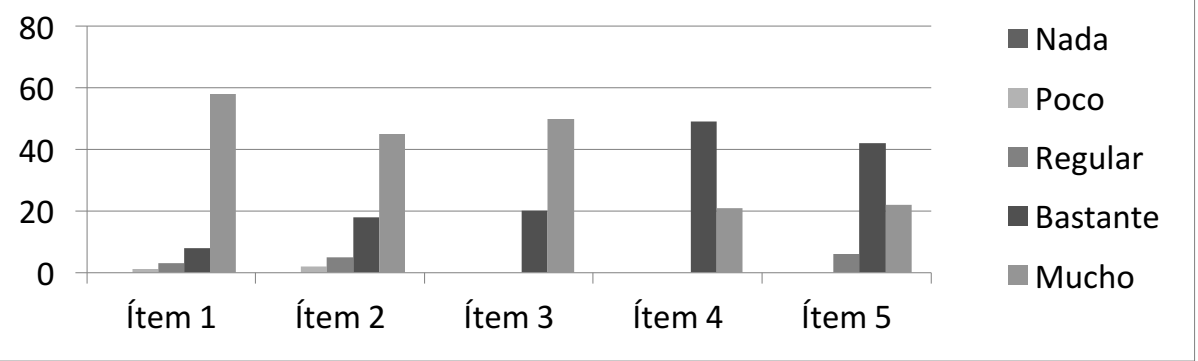

Figura 2. Resultados encuesta alumnado $4^{\circ} \mathrm{ESO}$ 
El vaciado nos permite observar el elevado grado de satisfacción del alumnado con el proyecto. Nos ha parecido destacable los resultados obtenidos en el ítem número cinco en la figura 2 que recoge los datos de los estudiantes de Secundaria, que muestra una opinión favorable respecto a su concepto de la lectura, teniendo en cuenta que, al iniciar el curso, en clase de literatura, le hicimos la misma pregunta y sus respuestas fueron que leer les parecía algo "aburrido", "pesado", "una pérdida de tiempo" o "cosa de frikis".

Del mismo modo, nos ha resultado gratificante la valoración positiva que hacen sobre el hecho de participar e interactuar con alumnos/as de diferentes edades, fundamentalmente por parte de los de Secundaria, ya que los de Primaria suelen tener a los grandes como un referente. Asimismo, nos parece remarcable el hecho de que valoren de manera tan positiva la dramatización teniendo en cuenta sus reticencias al presentarles el proyecto por miedo, por vergüenza y sentido del ridículo, inseguridades tan presentes en la adolescencia.

Finalmente, triangulando todos estos datos podemos deducir que se cumplen los objetivos

que nos hemos planteado y podemos hablar de unos resultados muy satisfactorios. Lo primordial ha sido lo bien que se lo han pasado los alumnos/as porque han aprendido disfrutando, haciendo, experimentado, probando, ensayando-errorando, (sobre todo en el caso de la dramatización y la interpretación, disciplinas por las que no están acostumbrados/as a transitar), destapando habilidades que desconocían tener, sorprendiéndose en cada etapa del proceso, expresando sus emociones, comprobando la importancia de involucrarse de manera individual y grupal, y descubriendo que los libros, la literatura, pueden ser una aventura fascinante. La motivación y la creatividad han ido in crescendo hasta darle forma al proyecto.

\section{Discusiones/Conclusiones}

Hemos podido constatar que, para la asignatura de Lengua castellana y Literatura, en este caso, se abre a un abanico de posibilidades donde lo inesperado y la sorpresa tienen cabida.

Teniendo siempre presente los contenidos y objetivos curriculares, hemos optado por diseñar dinámicas de aprendizaje con un nuevo enfoque didáctico desde el que trabajar la educación literaria. El trabajo del profesorado se convierte, de este modo, en un proceso de investigación y ensayo que busca actualizar la metodología, poner en práctica la interdisciplinariedad, desarrollar las competencias y adaptar el proceso de enseñanza-aprendizaje a las necesidades del siglo XXI.

El proyecto presentó varios desafíos: formar al alumnado en las estrategias de animación a la lectura, entusiasmarles con los libros escogidos, coordinación entre las diferentes áreas, organizar bien los grupos, ajustar el tiempo adecuadamente, atender a los diferentes grupos y un ejercicio de coordinación y trabajo que, en ocasiones, resulta desbordante.

Hubo cuestiones que pudieron resolver los propios alumnos/as: motivar a los/as más tímidos para la dramatización, investigar alternativas creativas para la creación de materiales e intercambiar ideas entre los diferentes grupos, lograr una buena coordinación y un adecuado reparto de tareas. También nos encontramos algunas dificultades: los alumnos/as comentaron que se vieron muy ajustados con el tiempo y sugirieron que se podía haber empezado antes. Estaban muy nerviosos/as el día de la dramatización, pero les encantó el entusiasmo con el que les recibieron los de Primaria.

En las entrevistas personales y en los debates de los talleres, sobre todo en el de la igualdad entre hombres y mujeres, muchos/as comentaron que no se habían percatado de la desigualdad existente aún en la sociedad y tampoco de determinadas ideas equivocadas con respecto a las relaciones de pareja, como, por ejemplo, muchos/as seguían viendo los celos como una demostración de amor. 
Finalmente, consideramos que el proyecto aporta beneficios reales y prácticos: desarrollo de las habilidades lingüísticas y de la capacidad crítica, acercamiento a los libros y una forma enriquecedora y diferente de conectar con la literatura.

\section{Referencias}

Abarca Alpízar, F. (2016). La metodología participativa para la intervención social: Reflexiones desde la práctica. Ensayos pedagógicos, 11 (1). Disponible en:

http://www.revistas.una.ac.cr/index.php/ensayospedagogicos/article/view/8470/9650

Acaso, M. (2014). Reduvolution. Hacer la revolución en la educación. Barcelona: Paidós.

Aguilar, L. (2008). Orejas de Mariposa. Madrid: Kalandraka.

Barthes, R. (2002). Variaciones sobre la literatura. Barcelona: Paidós.

Bécquer, G. A. (2006). Rimas. Madrid: Cátedra.

Real Decreto 1105/2014, de 26 de diciembre, por el que se establece el currículo básico de la Educación Secundaria Obligatoria y del Bachillerato. Boletín Oficial del Estado, núm. 3, de 3 de enero de 2015, pp. 169-546. Recuperada de https://www.boe.es/buscar/doc.php?id=BOEA-2015-37.

Broitman, A. I. (2015). La Estética de la Recepción. Bases teóricas para el análisis de las prácticas lectoras y otros consumos culturales. En I. Casanovas, M.G. Gómez, y E.J. Rico (eds.). Actas de la III Jornadas de investigación en edición, Cultura y Comunicación. Facultad de Filosofía y Letras de la Universidad de Buenos Aires, Buenos Aires.

Cole, B. (1990). La Princesa Listilla. Madrid: Destino.

Colomer, T. (2010). La didáctica de la literatura: temas y líneas de investigación e Innovación. Alicante: Biblioteca Virtual Miguel de Cervantes. Disponible en: http://www.cervantesvirtual.com/nd/ark:/59851/bmcpv725

Darío, R. (2002). Prosas Profanas y otros poemas. Barcelona: Espasa libros.

Dautremer, R. (2014). Enamorados. Madrid: Kokinos.

Eagleton, T. (2016). Cómo leer literatura. Barcelona: Península.

Enkvist, I. (2011). La buena y la mala educación. Madrid: Encuentro.

Mendoza Fillola, A. (2004). La educación literaria. Bases para la formación de la competencia lecto-literaria. Alicante: Biblioteca virtual Miguel de Cervantes. Disponible en: http://www.cervantesvirtual.com/obra/la-educacin-literaria---bases-para-la-formacin-de-lacompetencia-lectoliteraria-0/

Millás, J. J. (2016). Leer novelas fortalece el Aparato Imaginario. El País Digital. Disponible en: https://elpais.com/cultura/2016/08/22/actualidad/1471886325_438016.html?id_externo_r soc=TW_CC

O’Connor, F. (2016). Para escribir cuentos [Mensaje en un blog]. Disponible en: http://textosblog2013.blogspot.com.es/2013/11/el-arte-del-cuento-flannery-oconnor.html

Pennac, D. (1993). Como una novela. Barcelona: Anagrama.

Pérez Galdós, B. (2001). Marianela. Madrid: Anaya.

Rodari, G. (2002). Gramática de la fantasía. Madrid: Del Bronce.

Sánchez Calleja, L. y Sánchez Román, A. (2015). La educación emocional. Cuadernos de Pedagogía, (452), 86-91.

Servén Díez, C. (2008). Canon literario, educación y escritura femenina. OCNOS, (4), 7-20. 


\section{Información sobre los autores}

Autor: Blanca Hernández Quintana

Institución: Departamento de Didácticas Especiales. Área de Lengua y Literatura.

Universidad de Las Palmas de Gran Canaria

Email: blanca.hernandez@ulpgc.es 


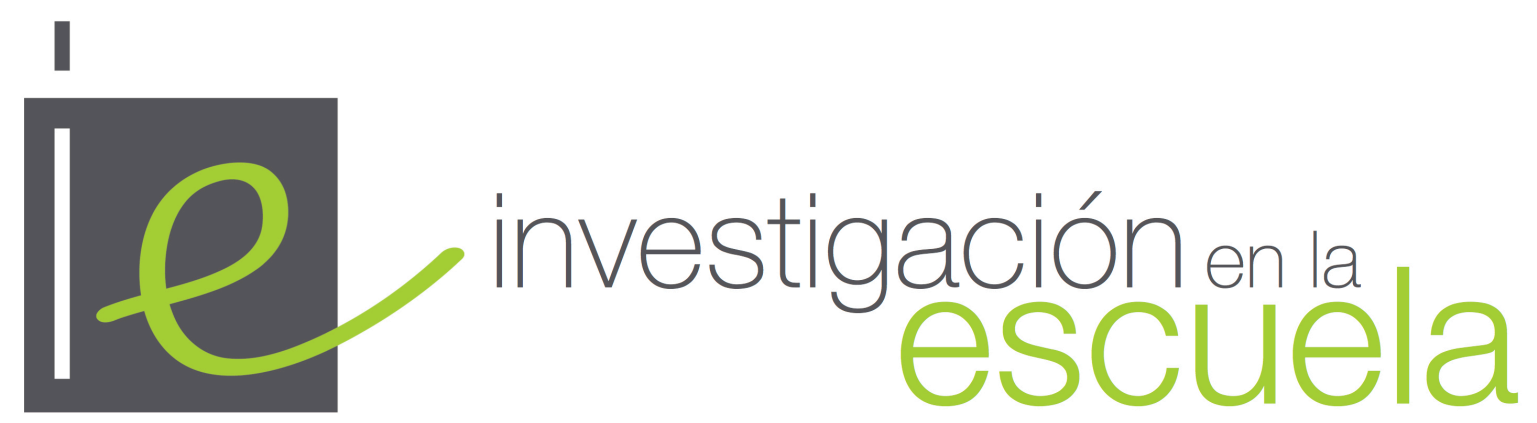

Revista académica evaluada por pares y de acceso abierto

Número 94

30 de abril de 2018

ISSN 2443-9991

\begin{abstract}
(c)
SOMEREIGHISRESEFVEDLOS/as lectores/as pueden copiar, mostrar, y distribuir este artículo, siempre y cuando se de crédito y atribución al autor/es y a Investigación en la Escuela, se distribuya con propósitos no-comerciales, no se altere o transforme el trabajo original. Más detalles de la licencia de CreativeCommons se encuentran en http://creativecommons.org/licenses/by-nc-sa/3.0 Cualquier otro uso debe ser aprobado en conjunto por el autor/es, o Investigación en la Escuela.
\end{abstract}

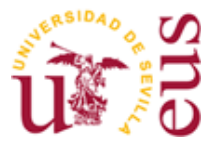
investigacion-en-la-escuela

Contribuya con comentarios y sugerencias en la web de la revista. Por errores y sugerencias contacteasecretaria@investigacionenlaescuela.es 


\section{Investigación en la escuela}

Consejo de dirección: Ana Rivero García (Universidad de Sevilla), Nicolás de Alba Fernández (Universidad de Sevilla), Pedro Cañal de León (Universidad de Sevilla), Francisco F. García Pérez (Universidad de Sevilla), Gabriel Travé González (Universidad de Huelva), Francisco F. Pozuelos Estrada (Universidad de Huelva)

Dirección: Ana Rivero García y Nicolás de Alba Fernández

Secretaría de edición: Elisa Navarro Medina

\section{Consejo editorial}

José Félix Angulo Rasco. Universidad de Cádiz Rosa Má Ávila Ruiz. Universidad de Sevilla Pilar AzcárateGoded. Universidad de Cádiz Juan Bautista Martínez Rodríguez. Universidad de Granada

Nieves Blanco García. Universidad de Málaga Fernando Barragán Medero. Universidad de La Laguna José Carrillo Yáñez. Universidad de Huelva José Contreras Domingo. Universidad de Barcelona. Luis C. Contreras González. Universidad de Huelva Ana $\mathbf{M}^{\mathbf{a}}$ Criado García-Legaz. Universidad de Sevilla Rosario Cubero Pérez. Universidad de Sevilla José $\mathbf{M}^{\mathbf{a}}$ Cuenca López. Universidad de Huelva Jesús Estepa Giménez. Universidad de Huelva Rafael Feito Alonso. Universidad Complutense (Madrid)

Francisco José García Gallardo. Universidad de Huelva

Soledad García Gómez. Universidad de Sevilla J. Eduardo García Díaz. Universidad de Sevilla
Fernando Hernández Hernández. Universidad de Barcelona

Salvador Llinares Ciscar. Universidad de Alicante Alfonso Luque Lozano. Universidad de Sevilla Rosa Martín del Pozo. Universidad Complutense (Madrid)

José Martín Toscano. IES Fernando Herrera (Sevilla) Jaume Martínez Bonafé. Universidad de Valencia F. Javier Merchán Iglesias. Universidad de Sevilla Emilia Moreno Sánchez. Universidad de Huelva. Rosario Ortega Ruiz. Universidad de Córdoba Antonio de Pro Bueno. Universidad de Murcia Fco. de Paula Rodríguez Miranda. Universidad de Huelva

Pedro Sáenz-López Buñuel. Universidad de Huelva Antoni Santisteban Fernández. Universidad Autónoma (Barcelona)

Emilio Solís Ramírez. Catedrático de IES. $\mathbf{M}^{\mathbf{a}}$ Victoria Sánchez García. Universidad de Sevilla. Magdalena Suárez Ortega. Universidad de Sevilla

\section{Consejo asesor}

Manuel Área Moreira. Universidad de La Laguna

Jaume Carbonell. Director Cuadernos de Pedagogía. Barcelona

César Coll. Universidad de Barcelona

Christopher Day. Universidad de Nothingham. U.K.

Juan Delval. Universidad Nacional de Educación a Distancia

John Elliott. Universidad de East Anglia. Norwich. U.K.

José Gimeno Sacristán. Universidad de Valencia

André Giordan. Universidad de Paris VII y Ginebra

Francisco Imbernón. Universidad de Barcelona

Ángel Pérez Gómez. Universidad de Málaga

Rafael Porlán Ariza. Universidad de Sevilla

Francesco Tonucci. Instituto de Pedagogía del C.N.R. Roma

Jurjo Torres Santomé. Universidad de A Coruña 
